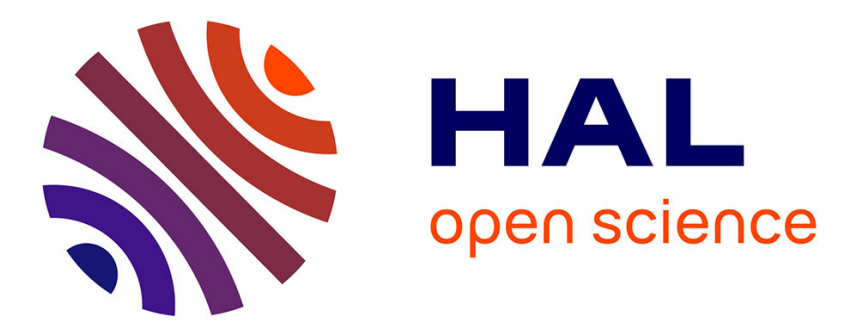

\title{
Noninvasive microbubble-based pressure measurements: a simulation study
}

\author{
Michiel Postema, Ayache Bouakaz, Nico de Jong
}

\section{To cite this version:}

Michiel Postema, Ayache Bouakaz, Nico de Jong. Noninvasive microbubble-based pressure measurements: a simulation study. Ultrasonics, 2004, Proceedings of Ultrasonics International 2003 (UI03) 30 June-3 July 2003, Granada, 42 (1-9), pp.759-762. 10.1016/j.ultras.2003.12.007 . hal-03193398

\section{HAL Id: hal-03193398 \\ https://hal.science/hal-03193398}

Submitted on 13 Apr 2021

HAL is a multi-disciplinary open access archive for the deposit and dissemination of scientific research documents, whether they are published or not. The documents may come from teaching and research institutions in France or abroad, or from public or private research centers.
L'archive ouverte pluridisciplinaire HAL, est destinée au dépôt et à la diffusion de documents scientifiques de niveau recherche, publiés ou non, émanant des établissements d'enseignement et de recherche français ou étrangers, des laboratoires publics ou privés.

\section{(ㅇ)(1) $\$$}

Distributed under a Creative Commons Attribution - NonCommercial - NoDerivatives| 4.0 


\title{
Noninvasive microbubble-based pressure measurements: a simulation study
}

\author{
Michiel Postema ${ }^{\mathrm{a}, \mathrm{b}, *}$, Ayache Bouakaz ${ }^{\mathrm{a}, \mathrm{b}}$, Nico de Jong ${ }^{\mathrm{a}, \mathrm{b}, \mathrm{c}}$ \\ ${ }^{a}$ Erasmus MC-University Medical Center, Thoraxcentre, Department of Experimental \\ Echocardiography, Dr. Molewaterplein 50, Rm. Ee-2302, 3000 DR Rotterdam, \\ The Netherlands \\ b Interuniversity Cardiology Institute of The Netherlands, Catharijnesingel 52, 3511 GC \\ Utrecht, The Netherlands \\ ${ }^{\mathrm{c}}$ University of Twente, Faculty of Science and Technology, Department of Applied \\ Physics, Physics of Fluids, P.O. Box 217, Rm. CTW N-200, 7500 AE Enschede, \\ The Netherlands
}

\begin{abstract}
This paper describes a noninvasive method to measure local hydrostatic pressures in fluid filled cavities. The method is based on the disappearance time of a gas bubble, as the disappearance time is related to the hydrostatic pressure. When a bubble shrinks, its response to ultrasound changes. From this response, the disappearance time, and with it the hydrostatic pressure, can be determined.

We investigated the applicability of the gases $\mathrm{Ar}, \mathrm{C}_{3} \mathrm{~F}_{8}, \mathrm{Kr}, \mathrm{N}_{2}$, $\mathrm{Ne}$, and $\mathrm{SF}_{6}$, based on their diffusive properties. For pressure measurements with a limited duration, e.g. $150 \mathrm{~ms}, \mathrm{Kr}$ and Ar bubbles are most suitable, since they are most sensitive to pressure change. If there is also a limitation to bubble size, e.g. a maximum diameter of $6 \mu \mathrm{m}, \mathrm{SF}_{6}$ is most suitable.

We present improvements of a method that correlates the duration of the decay of the fundamental ultrasound response to the hydrostatic overpressure. We propose to correlate the duration until subharmonic occurrence in combination with its decay, to hydrostatic overpressure, since the subharmonic decays more rapidly than the fundamental response. For a dissolving Ar gas bubble with an initial diameter of $14 \mu \mathrm{m}$, the overpressure can be determined 4 times as precise from the decay of the subharmonic response as from the decay of the fundamental response. Overpressures as small as $11 \mathrm{mmHg}$ may be discriminated with this method.
\end{abstract}

${ }^{*}$ Corresponding author. Address: Erasmus MC-University Medical Center, Thoraxcentre, Department of Experimental Echocardiography, Dr. Molewaterplein 50, Rm. Ee2302, 3000 DR Rotterdam, The Netherlands. Tel.: +31-10-4089358; fax: +31-10-4089445. E-mail address: m.postema@erasmusmc.nl (M. Postema). 


\section{Introduction}

Local pressure measurements in cavities are widely used in medical diag-

nostics. Local pressure measurements in the heart are generally done by catheterization, causing pain and risk of infection [1]. We propose a renewed noninvasive method to measure pressure in cavities, based on the diffusion of free gas microbubbles and their interaction with ultrasound waves.

Epstein \& Plesset [2] and De Jong et al. [3] demonstrated the relation between the disappearance time of gas bubbles and the hydrostatic pressure applied. Because the sizes of gas bubbles change as a function of the hydrostatic pressure, the acoustic properties of the bubbles are affected. Based on this finding, a relation between bubble disappearance time, its acoustic response, and ambient pressure can be established. Bouakaz et al. gave an overview of pressure measurement methods using this relation, and studied one in vitro [4]. Instead of free gas bubbles, they inserted ultrasound contrast agent in their setup. Hard-shelled ultrasound contrast agent can act as a vehicle to carry gas to a region of interest. Upon insonification at sufficient acoustic pressure, the gas is released. This process is called sonic cracking [5]. Bouakaz et al. determined overpressures from the decay of the fundamental acoustic response from diffusing released air bubbles. Pressure differences of $50 \mathrm{mmHg}$ could be distinguished theoretically and experimentally. In medical diagnostics a resolution lower than $50 \mathrm{mmHg}$ is desirable. To improve the sensitivity of the measurement approach mentioned above, we investigated 
the use of different gases. Furthermore, we investigated subharmonics as a marker for half resonant bubble size, since the subharmonic response is more sensitive to bubble size change than the fundamental [6].

Shi et al. had suggested the use of subharmonics for noninvasive pressure measurements [7]. They measured the scattering of encapsulated microbubbles over the pressure range $0-186 \mathrm{mmHg}$, and found a decrease of approximately $10 \mathrm{~dB}$. It had previously been demonstrated theoretically that subharmonic generation from free gas bubbles and from ultrasound contrast agents requires a threshold insonifying pressure, which is minimal when microspheres are insonified at twice their resonance frequency $[8,9]$. Palanchon et al. determined such thresholds with simulations and experiments for free microemboli [6].

Since subharmonics can be generated with the resonant bubble size corresponding to half the transmitted frequency, we propose to correlate the time until subharmonic occurrence, to the hydrostatic overpressure.

In this paper we present improvements of a previously published method that correlates the duration of the decay of the fundamental response to the hydrostatic overpressure [4]. To improve the method, we simulate the diffusive behavior of six gases, and suggest a qualitative measure for the applicability of a specific gas. We discuss the results with respect to boundary conditions and limitations, which hold for the ultimate in vivo situation. Furthermore, we simulate the scattering behavior of ultrasound-insonified diffusing gas bubbles. We investigate the sensitivity of the subharmonic 
response in comparison to the fundamental response.

\section{Methods}

\subsection{Theoretical model}

The change of gas bubble radius as a function of time, is given by [3, 4]:

$$
\frac{\mathrm{d} R}{\mathrm{~d} t}=D L\left(\frac{\frac{C_{\mathrm{i}}}{C_{0}}-1-\frac{2 \sigma}{R p_{0}}-\frac{p_{\mathrm{ov}}}{p_{0}}}{1+\frac{4 \sigma}{3 R p_{0}}}\right)\left(\frac{1}{R}+\frac{1}{\sqrt{\pi D t}}\right),
$$

where $\frac{C_{\mathrm{i}}}{C_{0}}$ is the ratio of the dissolved gas concentration to the saturation concentration (saturation ratio), $D$ is the diffusion constant, $L$ is the Ostwald coefficient, $p_{0}$ is the ambient pressure, $p_{\text {ov }}$ is the applied overpressure, $R$ is the instantaneous bubble radius, $t$ is the time starting $(t=0)$ when the bubble surface is exposed to the liquid surface, and $\sigma$ is the surface tension. Eq. (1) shows that the disappearance of gas bubbles in a liquid medium is highly influenced by gas diffusion parameters and applied overpressure, and that the disappearance time of gas bubbles is shorter when the liquid medium is under pressure.

When a gas bubble dissolves into a liquid medium, its acoustic response changes with its radius. The oscillating behavior of a gas bubble in a liquid, subjected to a sound field with a low acoustic pressure, was derived by [10]. The changes in oscillating behavior of the dissolving gas bubble lead 
to changes in scattering cross sections [11], and thus, the scattering behavior of an insonified, diffusing gas bubble can be calculated, dependent of the applied overpressure.

\subsection{Simulations}

First, we investigate the diffusive behavior of six gases by simulations, and define a qualitative measure for the applicability of a specific gas. Then we explore the sensitivity of the subharmonic acoustic response from diffusing gas bubbles, in comparison to the fundamental response.

The disappearance of free gas bubbles was simulated at hydrostatic overpressures between 0 and $200 \mathrm{mmHg}$, with gases $\mathrm{Ar}, \mathrm{C}_{3} \mathrm{~F}_{8}, \mathrm{Kr}, \mathrm{N}_{2}, \mathrm{Ne}$, and $\mathrm{SF}_{6}$. For our computations we used MatraB ${ }^{\circledR}$ (The MathWorks, Inc., Natick, MA) programs. The parameters used were published in [12]. The diffusion constants and the Ostwald coefficients were used for gas diffusing in

water at room temperature $[13,14,15,16,17]$. Saturation ratios $\frac{C_{\mathrm{i}}}{C_{0}}=0$ were used, indicating that the gases are not present in water. From the diffusion curves, we computed the times it takes for bubbles to diffuse until they reach half their initial diameters. Half-size times were computed for gas bubbles varying in diameters from 5 to $20 \mu \mathrm{m}$, at ambient pressure, and at $20 \mathrm{mmHg}$ overpressure. When applying an overpressure, the half-size time of a bubble is shorter than the half-size time at ambient pressure, $t_{1_{2}}$. This difference in half-size times, $\Delta t_{1 / 2}$, is a qualitative measure for the applicability of a specific gas for the measurement of hydrostatic overpressures: The sensitivity of the 
bubble to pressure change improves when $\Delta t_{1 / 2}$ increases.

Scattering cross-sections were calculated for the diffusing gas bubbles as a function of time $[10,11,12]$, after filtering the fundamental and subharmonic responses from the acoustic bubble responses using a band-pass filter. The acoustic frequencies simulated, ranged from $0.5 \mathrm{MHz}$ to $10 \mathrm{MHz}$.

\section{Results and discussion}

From our simulations it follows that diffusion duration, and with it $\Delta t_{y_{2}}$, increases with the initial bubble diameter $\varnothing$. However, bubble size is a limiting factor in the in vivo situation, as an encapsulated bubble may have to pass through narrow vessels before arriving in the cavity where the gas is released. The measurement duration is another limitation, especially for blood pressure measurements, since pressure changes occur within the cardiac

cycle. A measurement of the systolic pressure in the left ventricle is limited to a duration of roughly $150 \mathrm{~ms}$ [18].

Table 1 gives an overview of values $\Delta t_{y_{2}}$, for different limitations. If the half-size time is limited to $150 \mathrm{~ms}$, and there is no limit to the bubble size, $\mathrm{Kr}$ and $\mathrm{Ar}$ gas bubbles result in the highest $\Delta t_{1_{2}}$. If the bubble diameter is limited to $6 \mu \mathrm{m}$, and there is no limit to the measurement duration, $\mathrm{C}_{3} \mathrm{~F}_{8}$ and $\mathrm{SF}_{6}$ bubbles result in the highest $\Delta t_{1_{2}}$. If both limitations are combined, $\mathrm{SF}_{6}$ and $\mathrm{N}_{2}$ bubbles result in the highest $\Delta t_{y_{2}}$. Hence, the applicability of a specific gas is mainly determined by the limitations that apply. 


\begin{tabular}{|c|c|c|c|c|c|c|}
\hline \multirow[t]{3}{*}{ Gas } & \multicolumn{6}{|c|}{ Limitation } \\
\hline & \multicolumn{2}{|c|}{$t_{1 / 2} \leqslant 150 \mathrm{~ms}$} & \multicolumn{2}{|c|}{$\varnothing \leqslant 6 \mu \mathrm{m}$} & \multicolumn{2}{|c|}{$\begin{array}{l}t_{1 / 2} \leqslant 150 \mathrm{~ms} \wedge \\
\varnothing \leqslant 6 \mu \mathrm{m}\end{array}$} \\
\hline & $\begin{array}{c}\varnothing \\
(\mu \mathrm{m})\end{array}$ & $\begin{array}{l}\Delta t_{1 / 2} \\
(\mathrm{~ms})\end{array}$ & $\begin{array}{r}t_{1 / 2} \\
(\mathrm{~ms})\end{array}$ & $\begin{array}{l}\Delta t_{1 / 2} \\
(\mathrm{~ms})\end{array}$ & $\begin{array}{c}\varnothing \\
(\mu \mathrm{m})\end{array}$ & $\begin{array}{l}\Delta t_{1 / 2} \\
(\mathrm{~ms})\end{array}$ \\
\hline $\mathrm{Ar}$ & 12 & 3.1 & 35 & 0.6 & 6.0 & 0.6 \\
\hline $\mathrm{C}_{3} \mathrm{~F}_{8}$ & 1.0 & 0.8 & $>1000$ & $>12$ & 1.0 & 0.8 \\
\hline $\mathrm{Kr}$ & 14 & 3.2 & 24 & 0.4 & 6.0 & 0.4 \\
\hline $\mathrm{N}_{2}$ & 7.5 & 2.8 & 99 & 1.6 & 6.0 & 1.6 \\
\hline $\mathrm{Ne}$ & 8.5 & 2.7 & 72 & 1.2 & 6.0 & 1.2 \\
\hline $\mathrm{SF}_{6}$ & 3.5 & 1.7 & 453 & 7.2 & 3.5 & 1.7 \\
\hline
\end{tabular}

Table 1: Half-size time differences for different limitations.

Shi et al. noted, that yet another limitation of our approach may lie in the fact that the disappearance times of the gas bubbles depend also on the gas content of the blood [7]. We may overcome this limitation by choosing gases that are not present in the human body, and as such have a saturation ratio $\frac{C_{\mathrm{i}}}{C_{0}}=0$.

Figure 1 shows the fundamental and subharmonic scattered power of a dissolving $\oslash 14 \mu \mathrm{m}$ Ar bubble, insonified at 1 and $2 \mathrm{MHz}$, respectively, as a function of time. The solid lines represent the situation at ambient pressure, the dashed at a $50 \mathrm{mmHg}$ hydrostatic overpressure. The maximum of the fundamental is reached gradually, whereas the subharmonic has a rapid rise near double-resonant size. The subharmonic peaks decay with $40 \mathrm{~dB}$ in $8.2 \mathrm{~ms}$, whereas the most rapid fundamental decay is only $20 \mathrm{~dB}$ in $15.2 \mathrm{~ms}$. Hence, the subharmonic response is more sensitive to diameter change than the fundamental response, indeed. Since overpressures of $50 \mathrm{mmHg}$ could be distinguished from the decay of the fundamental response [4], we estimate 


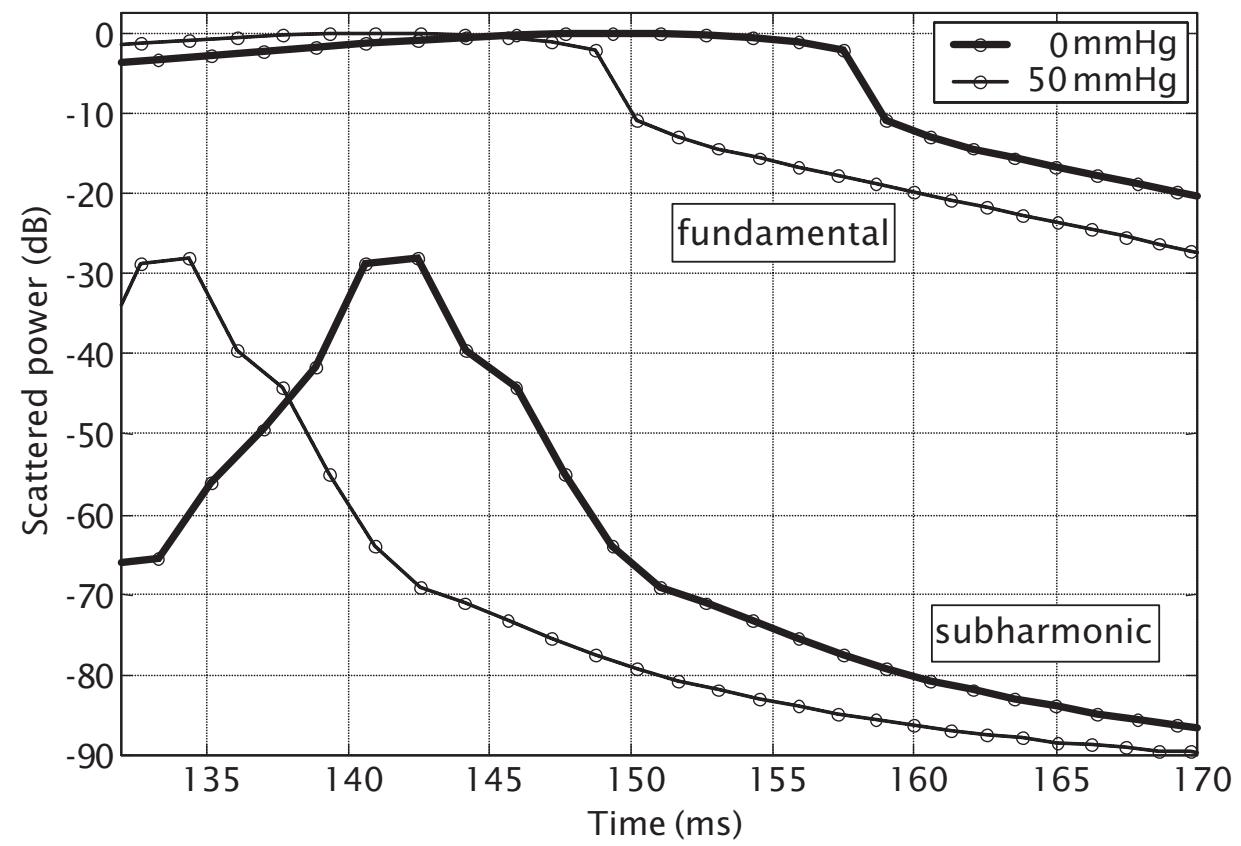

Figure 1: Fundamental and subharmonic scattering cross-sections of a diffusing $\oslash 14 \mu \mathrm{m}$ Ar bubble, insonified at 1 and $2 \mathrm{MHz}$, respectively, as a function of time, when applying hydrostatic overpressures of 0 and $50 \mathrm{mmHg}$.

that the scattering may have a $\pm 5 \mathrm{~dB}$ variation. With this variation, we computed diffusion time differences $\Delta t_{\mathrm{d}}$ for the decays observed in Figure 1: $\Delta t_{\mathrm{d}}=7.6 \mathrm{~ms}$ for the fundamental response, and $\Delta t_{\mathrm{d}}=2.0 \mathrm{~ms}$ for the subharmonic response. In our simulations, these diffusion time differences correspond to hydrostatic overpressures of 45 and $11 \mathrm{mmHg}$, respectively. Hence, the overpressure can be determined 4 times as precisely from the decay of the subharmonic response as from the decay of the fundamental response.

As an improvement of noninvasive pressure measurements, we propose to correlate the duration until the subharmonic peak in combination with the 
subharmonic decay, to hydrostatic overpressure. Evidently, precise knowledge of the initial bubble size is of importance. This might be established by generating subharmonics around the initial bubble size too.

Controlled gas release from a single bubble is currently under investigation in vitro with an ultrafast framing camera system [19].

\section{Conclusions}

For pressure measurements with a limited duration, e.g. $150 \mathrm{~ms}$, Kr and Ar bubbles are most suitable, since they are most sensitive to pressure change. If there is also a limitation to bubble size, e.g. a maximum diameter of $6 \mu \mathrm{m}$, $\mathrm{SF}_{6}$ is most suitable.

When a diffusing gas bubble with known initial diameter is insonified, the duration until subharmonic occurrence in combination with its decay, is an indicator of the hydrostatic overpressure. The subharmonic decays more rapidly than the fundamental response. For a diffusing Ar gas bubble with an initial diameter of $14 \mu \mathrm{m}$, the overpressure can be determined 4 times as precisely from the decay of the subharmonic response as from the decay of the fundamental response. Overpressures as small as $11 \mathrm{mmHg}$ may be discriminated with this method. Generating subharmonics may also be useful for verifying the initial bubble size.

Free gas bubbles can be delivered to cavities, and released by means of sonic cracking, which is currently under investigation. 


\section{Acknowledgements}

This work has been supported by the Technology Foundation STW (RKG.5104).

\section{References}

[1] C.L. Sprung (Ed.), Pulmonalarterienkatheter: Methodik und klinische Anwendung, 2nd ed. Springer-Verlag, Berlin, 1999.

[2] P.S. Epstein, M.S. Plesset, On the stability of gas bubbles in liquid-gas solutions, J. Chem. Phys., 18 (11) (1950) 1505-1509.

[3] N. de Jong, F.J. Ten Cate, C.T. Lancée, J.R.T.C. Roelandt, N. Bom, Principles and recent developments in ultrasound contrast agents, Ultrasonics, 29 (1991) 324-330.

[4] A. Bouakaz, P.J.A. Frinking, N. de Jong, N. Bom, Noninvasive measurement of the hydrostatic pressure in a fluid-filled cavity based on the disappearance time of micrometer-sized free gas bubbles, Ultrasound Med. Biol., 25 (9) (1999) 1407-1415.

[5] M. Postema, A. Bouakaz, C.T. Chin, N. de Jong, Optically observed microbubble coalescence and collapse, Proc. IEEE Ultrason. Symp., München, 2002, pp. 1900-1903. 
[6] P. Palanchon, A. Bouakaz, J. Klein, N. de Jong, Subharmonic and ultraharmonic emissions for emboli detection and characterization, Ultrasound Med. Biol., 29 (3) (2003) 417-425.

[7] W.T. Shi, F. Forsberg, J.S. Raichlen, Pressure measurements using contrast microbubbles, in: B.B. Goldberg, J.S. Raichlen, F. Forsberg (Eds.), Ultrasound Contrast Agents. Basic principles and clinical applications, second ed., London: Martin Dunitz, London, 2001, pp. 105-111.

[8] A. Prosperetti, Application of the subharmonic threshold to the measurement of the damping of oscillating gas bubbles, J. Acoust. Soc. Am., 61 (1) (1977) 11-16.

[9] P.M. Shankar, P.D. Krishna, V.L. Newhouse, Subharmonic backscattering from ultrasound contrast agents, J. Acoust. Soc. Am., 106 (4) (1999) 2104-2110.

[10] K.E. Morgan, J.S. Allen, P.A. Dayton, J.E. Chomas, A.L. Klibanov, K.W. Ferrara, Experimental and theoretical evaluation of microbubble behavior: Effect of transmitted phase and bubble size, IEEE Trans. Ultrason., Ferroelect., Freq. Contr., 47 (6) (2000) 1494-1509.

[11] S. Hilgenfeldt, D. Lohse, M. Zomack, Sound scattering and localized heat deposition of pulse-driven microbubbles, J. Acoust. Soc. Am., 107 (6) (2000) 3530-3539. 
[12] N. de Jong, R. Cornet, C.T. Lancée, Higher harmonics of vibrating gasfilled microspheres. Part one: simulations, Ultrasonics, 32 (6) (1994) $447-453$.

[13] E. Wilhelm, R. Battino, R.J. Wilcock, Low-pressure solubility of gases in liquid water, Chem. Rev., 77 (2) (1977) 219-262.

[14] D.B. King, E.S. Saltzman, Measurement of the diffusion coefficient of sulfur hexafluoride in water, J. Geophys. Res., 100 (C4), (1995) 70837088.

[15] J.E. Chomas, P. Dayton, J. Allen, K. Morgan K.W. Ferrara, Mechanisms of contrast agent destruction, IEEE Trans. Ultrason., Ferroelect., Freq. Contr., 48 (1) (2001) 232-248.

[16] D.R. Lide, CRC Handbook of Chemistry and Physics, 82 ed., CRC Press LLC, Boca Raton, FL, 2001.

[17] S. Mayer, P.A. Grayburn, Myocardial contrast agents: recent advances and future directions, Prog. Cardiovasc. Dis., 44 (1) (2001) 33-44.

[18] R.M. Berne, M.N. Levy (Eds.), Physiology, third ed., Mosby-Year Book, Mosby, St. Louis, 1993.

[19] C.T. Chin, C. Lancée, J. Borsboom, F. Mastik, M. Versluis, D. Lohse, N. de Jong, Optical imaging of ultrasound contrast bubble motions at 25 million frames per second, Abstr. 8th Eur. Symp. Ultrasound Contrast Imaging, Rotterdam, 2003, pp. 20-25. 